\title{
Delayed Leukoencephalopathy after Carbon Monoxide Poisoning Presenting as Subacute Dementia
}

\author{
Yu Mizuno ${ }^{1}$, Yasuhisa Sakurai ${ }^{1}$, Izumi Sugimoto ${ }^{1}$, Keiko Ichinose ${ }^{2}$, Shoichiro Ishihara ${ }^{2}$, \\ Nobuo Sanjo ${ }^{2}$, Hidehiro Mizusawa ${ }^{2}$ and Toru Mannen ${ }^{1}$
}

\begin{abstract}
We herein report the case of a 65-year-old woman who presented with the subacute onset of dementia and subsequently developed abnormal behavior and a gait disturbance. Her condition transiently improved; however, within one month, she became drowsy and poorly responsive, with limb chorea and urinary incontinence. Her history of frequently using charcoal led us to diagnose her with carbon monoxide (CO) poisoning. The findings of this case and a literature review suggest that subacute dementia due to CO poisoning recovers late, after a year or more, in patients above sixty years of age, and both hyperbaric oxygen and corticosteroid pulse therapy should be considered in such cases, even after one month.
\end{abstract}

Key words: carbon monoxide poisoning, delayed leukoencephalopathy, subacute dementia

(Intern Med 53: 1441-1445, 2014)

(DOI: 10.2169/internalmedicine.53.2132)

\section{Introduction}

Carbon monoxide $(\mathrm{CO})$ poisoning often presents as delayed encephalopathy in which neurological sequelae develop two to 40 days after an apparent recovery from acute cognitive deterioration that lasts 24 to 48 hours (1). The acute symptoms include headache, nausea, confusion and coma, depending on the blood carboxyhemoglobin $(\mathrm{CO} \mathrm{Hb})$ level, whereas delayed encephalopathy involves parkinsonism, incontinence, dementia and psychosis. The diagnosis is made based on known $\mathrm{CO}$ exposure and the blood $\mathrm{CO} \mathrm{Hb}$ level (2).

However, problems with the diagnosis arise when there is no such evidence of exposure. In addition, how should physicians establish the diagnosis of $\mathrm{CO}$ poisoning in cases in which the patient does not lapse into an acute coma? We herein report the case of a patient with suspected $\mathrm{CO}$ exposure who exhibited the subacute onset of dementia and review the clinical course and treatment of such patients in the literature.

\section{Case Report}

A 65-year-old woman was referred to our hospital in March due to a low-grade fever lasting for two days with the subsequent development of abnormal behavior (she drank her husband's alcohol, despite not having a drinking habit), dysarthria, lack of initiative (she stopped doing household chores) and a stooped posture while walking for over one month. She visited a local hospital ten days after disease onset and was given an intravenous drip infusion of vitamin $B_{1}$ for four days. Her condition immediately improved; however, she later developed urinary incontinence 14 days after onset. Her gait again worsened, and she required a wheelchair when leaving the house.

On the first examination, the patient was drowsy and poorly responsive and showed forced grasping with chorea of all four limbs. She did not obey one-step commands. Her lower limbs were spastic, with an increased left Achilles tendon reflex. MRI performed 13 days after disease onset revealed bilateral symmetric hyperintensity in the subcortical white matter of the basal frontal and parahippocampal gyri, periventricular white matter and centrum semiovale (Fig. 1, upper). She was admitted to our hospital with a diagnosis of

${ }^{1}$ Department of Neurology, Mitsui Memorial Hospital, Japan and ${ }^{2}$ Department of Neurology, Tokyo Medical and Dental University, Japan Received for publication November 14, 2013; Accepted for publication January 6, 2014

Correspondence to Dr. Yasuhisa Sakurai, ysakurai-tky@umin.ac.jp 

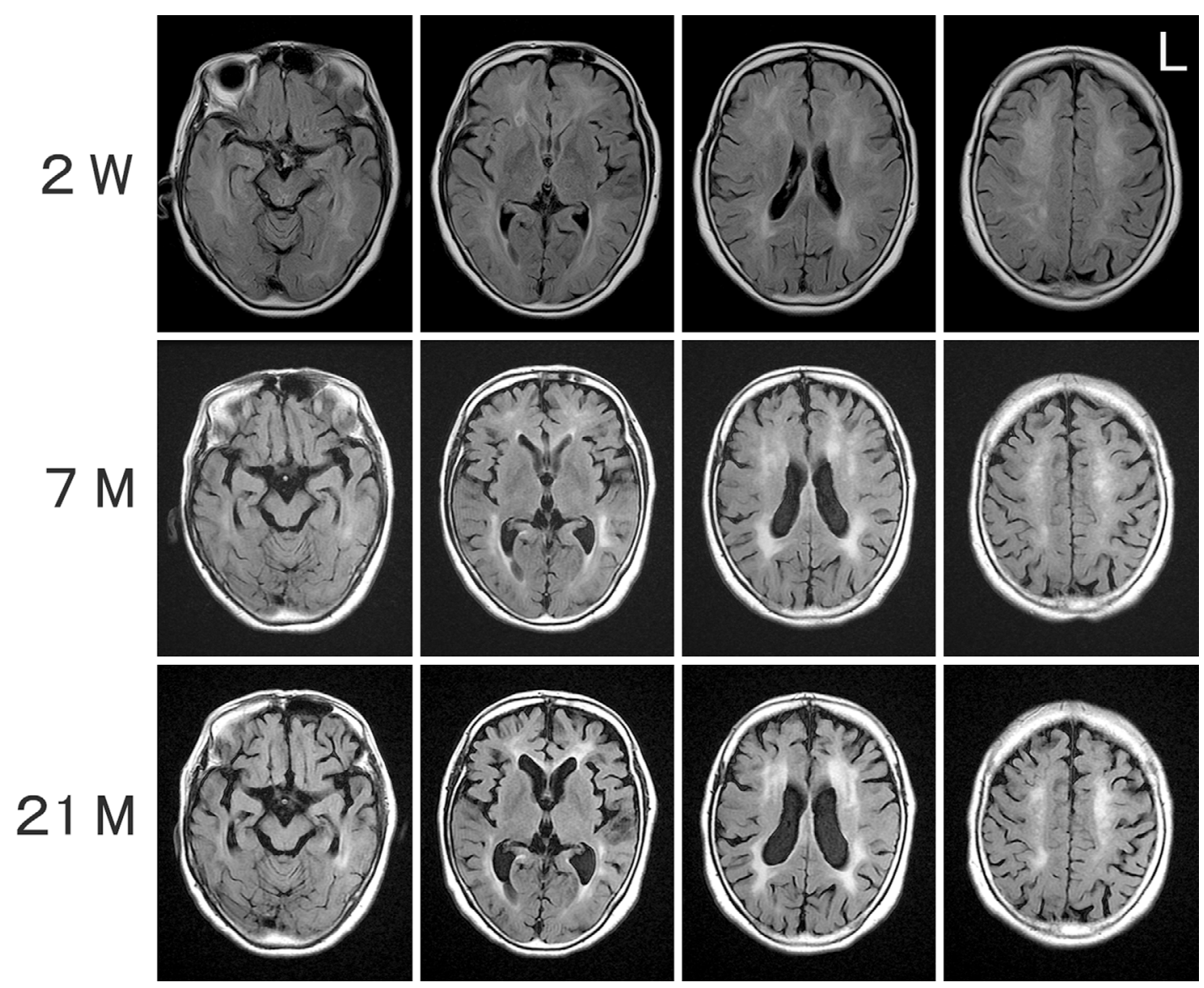

Figure 1. MR images of the patient. Fluid-attenuated inversion recovery (FLAIR) axial images obtained two weeks after onset (upper) revealed bilateral symmetric hyperintensity in the subcortical white matter in the basal frontal and parahippocampal gyri, periventricular white matter and centrum semiovale. On FLAIR axial images obtained seven months after onset (middle), the periventricular and deep white matter hyperintense regions remained, although they had decreased in size. However, the lateral ventricles had slightly enlarged. At 21 months after onset (lower), the ventricular enlargement had progressed further. $2 \mathrm{~W}$ : two weeks after onset, $7 \mathrm{M}$ : seven months after onset, $21 \mathrm{M}$ : 21 months after onset

leukoencephalopathy of an unknown etiology. The chorea worsened, and she was restless in the evening, necessitating frequent sedation with an intravenous drip infusion of haloperidol for 10 days after admission. Over the course of one week, the chorea remained limited to the lower limbs. The patient exhibited echolalia in response to a doctor's questions, as well as environmental dependency behavior, e.g., trying to place a toothbrush in her mouth upon seeing it. The laboratory data disclosed elevated serum lactate dehydrogenase (LDH) (253 U/L) and soluble IL2-receptor (281 $\mathrm{IU} / \mathrm{mL})$ levels; however, the C-reactive protein level $(0.1$ $\mathrm{mg} / \mathrm{dL})$ and white blood cell count $(6,000 / \mu \mathrm{L})$ were normal. A cerebrospinal fluid (CSF) analysis showed a normal cell count of $1 / \mu \mathrm{L}$ and a total protein level of $14 \mathrm{mg} / \mathrm{dL}$, with an elevated myelin basic protein level $(567 \mathrm{pg} / \mathrm{mL})$ and $\mathrm{IgG}$ index $(0.85$, normal <0.7), calculated according to the formula: (CSF-IgG/CSF-albumin)/(serum IgG/serum albumin). Antibodies associated with paraneoplastic neurological syndromes, i.e., Ma2, Ma1, amphiphysin, CV2, Ri, Yo, HuD and anti-N-methyl-D-aspartate-receptor (NMDAR), as well as herpes simplex virus (HSV)- $\operatorname{IgM}$, herpes zoster virus (HZV)-IgM and HIV antibodies, were all negative.

Detailed history-taking from the patient's husband revealed that they had used charcoal at home for heating and boiling water for a private samisen (Japanese traditional lute) lesson for four or five hours a day from November of the previous year to February (four months). The patient had always made the fire. Therefore, it is possible that she had unknowingly been exposed to low levels of CO. Although the patient's husband and students may also have inhaled low-dose $\mathrm{CO}$, they did not show any symptoms of $\mathrm{CO}$ intoxication (e.g., headache) because they frequently opened the door for air ventilation.

The possible $\mathrm{CO}$ exposure, together with characteristic biphasic consciousness disturbances and symmetric leukoencephalopathy on MRI, strongly suggested CO poisoning. The patient was administered intravenous corticosteroid pulse therapy (methylprednisolone, $1,000 \mathrm{mg}$ a day) for three days starting at 51 days post-onset. Subsequently, the echolalia and chorea mostly disappeared. However, she was 


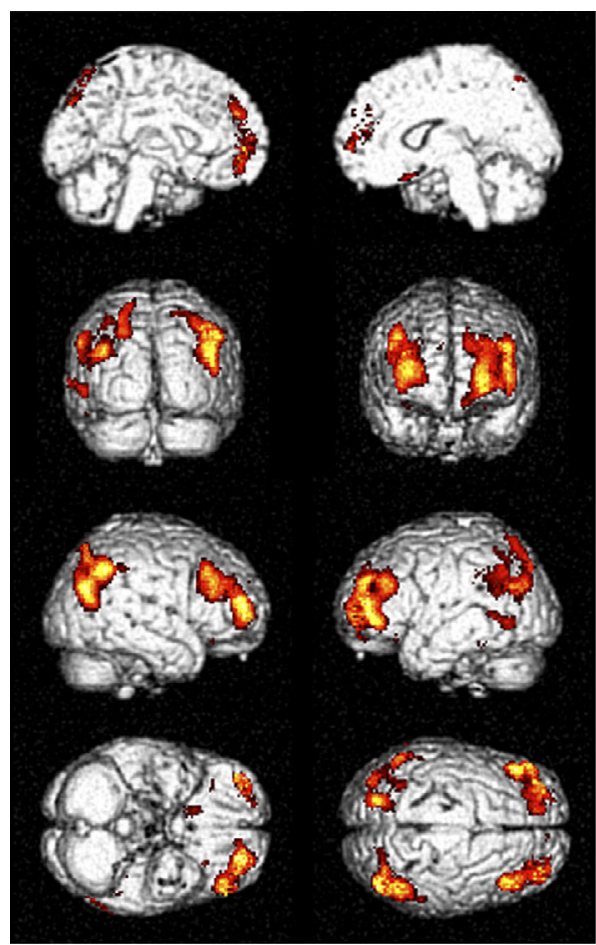

$4 \mathrm{M}$

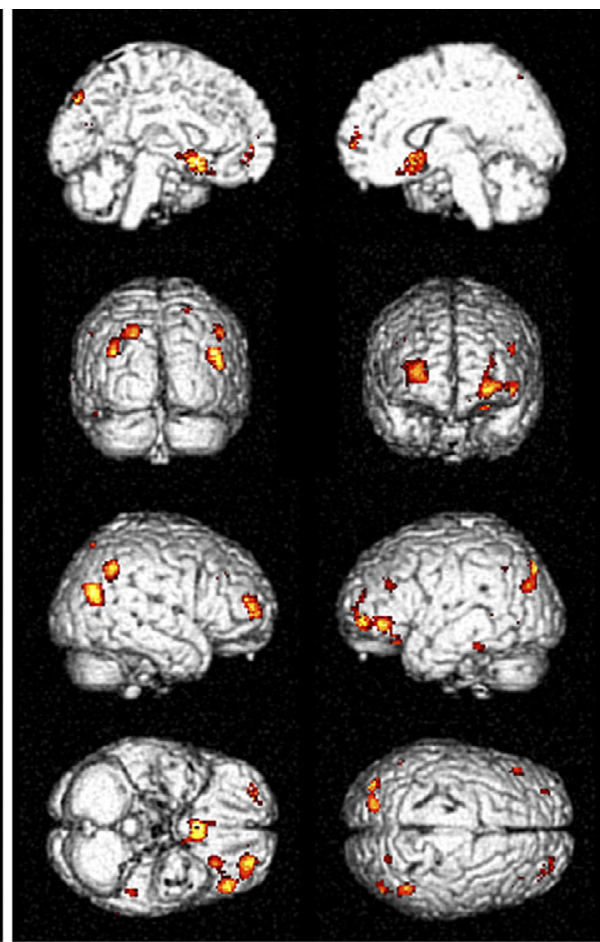

$1 \mathrm{yr}$

Figure 2. ${ }^{99} \mathrm{~m}$ Tc-ECD-SPECT images obtained at four months and one year after onset. The SPECT data were transformed into the Analyze format and normalized, smoothed and corrected for interlaboratory differences with a three-dimensional conversion map using the easy $Z \mathbf{Z}$ score imaging system (eZIS) (14) version 3. The images were then compared with those of a normal subject database of the same generation and gender $(n=22)$ with a two-sample t-test of Statistical Parametric Mapping (SPM) 2 (15) to show areas with a significant decrease in blood flow (uncorrected $\mathbf{p}<0.001$ ) on standard brain surface images. The SPECT images obtained four months after onset (left) revealed hypoperfusion in the bilateral frontal convexity and mesial frontal and temporoparietal areas. The regions with hypoperfusion had markedly reduced in size at one year after onset (right). 4M: four months after onset, 1yr: one year after onset

euphoric, persisting in fiddling with a blanket continuously with her right hand (frontal alien hand sign) (3) despite a doctor talking to her (attentional disorder), stirring an empty cup with chopsticks when presented with the cup and chopsticks (compulsive manipulation of tools) (4) and continuing to show forced grasping. She was able to read aloud her kana (Japanese phonograms) name written on a sheet of paper; however, when asked to write her name, she wrote lines repeatedly (motor perseveration).

The patient was transferred to the Department of Neurology at the Medical Hospital of Tokyo Medical and Dental University, Tokyo for hyperbaric oxygen therapy. She was given a total of 19 oxygen therapy sessions during hospitalization for one month; however, she still wandered at night. She was then transferred again to our hospital for a reevaluation. She required sedation with quetiapine for four months after onset, when she became relatively independent in her activities of daily living.

The Mini-Mental State Examination (MMSE) conducted four months after onset for the first time during hospitaliza- tion revealed moderate cognitive impairment (11.4/30), consisting of disorientation of time and space, agraphia [perseveration and neographism of kanji (Japanese morphograms) characters], acalculia, recent memory impairment (the 5-minute delayed three-word reproduction score was 0/ 3). The Frontal Assessment Battery (FAB), conducted at the same time, showed a severe dysexecutive function $(6 / 18$; word fluency, 1 word). The patient's digit span forward score on the Wechsler Adult Intelligence Scale-III (WAISIII) was three. Single photon emission computed tomography with a ${ }^{99 m}$ Tc-ethylcysteinate dimer (ECD-SPECT) performed four months after onset revealed hypoperfusion in the bilateral frontal convexity and mesial frontal and temporoparietal areas (Fig. 2). Electroencephalogram (EEG) performed during this period showed a small amount of 8-9$\mathrm{Hz} \alpha$ waves in F-C-P-O, which contrasted markedly with that of the moderate amount of $4-\mathrm{Hz} \theta$ waves with occasional $2-3-\mathrm{Hz} \delta$ waves in Fp-C-O observed at onset.

After discharge, the patient became independent in activities of daily living; the Barthel Index improved from 25 at 
Table. Patients with CO Poisoning Presenting as Subacute Dementia

\begin{tabular}{lccll}
\hline Reference & Patient & \multicolumn{2}{c}{ Treatment } & Outcome \\
& & HBO (time p.o./counts) & PSL (time p.o.) \\
\hline$(5)$ & $48 \mathrm{~W}$ & n.d. & 12 days* & 90 days, recovery \\
$(6)$ & $45 \mathrm{~W}$ & 50 days/6 & n.d. & 60 days, recovery \\
$(7)$ & $57 \mathrm{M}$ & 30 days/15 & n.d. & 90 days, recovery \\
$(8)$ & $52 \mathrm{~W}$ & n.d. & n.d. & 120 days, HDS-R 17/30 \\
$(9)$ & $75 \mathrm{~W}$ & 15 days/50 & n.d. & 80 days, disorientation \\
$(10)$ & $67 \mathrm{~W}$ & 40 days/3 Mos & n.d. & 5 Mos, HDS-R 20/30 \\
Our patient & $65 \mathrm{~W}$ & 51 days/19 & 37 days\# & 4 Mos, MMSE 11.4/30 \\
& & & & 14 Mos, MMSE 27.7/30 \\
\hline
\end{tabular}

HBO: Hyperbaric oxygen therapy, PSL: Prednisolone administration, W: Woman, M:

Man, p.o.: Post-onset, Mos: Months, n.d.: Not done, HDS-R: Hasegawa Dementia Scale

Revised, MMSE: Mini-Mental State Examination

*After corticosteroid pulse therapy (methylprednisolone, at $1,000 \mathrm{mg} /$ day for three

days), oral prednisolone (initial dose, $60 \mathrm{mg}$ /day) was administered for ten days.

\#Methylprednisolone 1,000-mg pulse therapy for three days only

one month after onset to 100 at six months after onset. Follow-up MRI performed seven months after onset showed that the periventricular and deep white matter hyperintense regions remained, although they had decreased in size (Fig. 1, middle). However, the lateral ventricles had become slightly enlarged, and the ventricular enlargement was found to have progressed further on MRI performed one year and nine months after onset (Fig. 1, lower). ECD-SPECT performed one year after onset revealed that the regions with hypoperfusion in the bilateral frontal and parietal areas had markedly reduced in size (Fig. 2). The MMSE score improved to the normal range (27.7/30) and the digit span forward score was four at 14 months after onset, although the FAB score remained low (14/18), even at 18 months after onset.

\section{Discussion}

The present patient presented with the subacute progression of dementia lasting for one month. We diagnosed her with delayed leukoencephalopathy due to $\mathrm{CO}$ poisoning based on the facts that: i) she had used charcoal for four to five hours a day over a period of four months and was believed to have frequently been exposed to low levels of $\mathrm{CO}$ during that period; ii) biphasic consciousness disturbances occurred; and iii) MRI showed symmetric leukoencephalopathy.

The delayed neurological sequelae observed after 40 days in this case included dementia with nocturnal delirium and dysexecutive syndrome characterized by environmental dependency behavior, the alien hand sign and the compulsive manipulation of tools. Four months later, when we were able to first evaluate the patient's cognitive function, she exhibited agraphia, acalculia, reduced verbal short-term memory and recent memory impairment. These findings were consistent with the ECD-SPECT results obtained at the same time, which revealed hypoperfusion in the bilateral dorsolateral frontal, mesial frontal and temporoparietal areas. She became independent in activities of daily living within six months, and her cognitive function improved to a nearly normal level, except for the executive function, within 14 months, in accordance with the reduction of high-intensity white matter regions on MRI and areas with hypoperfusion on SPECT.

The patient did not develop an acute-phase comatose state and exhibited subacute dementia a few days to weeks after exposure to $\mathrm{CO}$. Subacute dementia due to $\mathrm{CO}$ poisoning, lacking an acute coma, is a rare disorder. A MEDLINE and ICHUSHI (Japan Medical Abstracts Society) search for articles published between January 1960 and September 2013 was conducted using the key words "CO poisoning" and "dementia." Among the obtained articles, we selected cases with subacute (progression more than one week to as long as six weeks) dementia without witnessed acute coma. The table shows these reported cases. All patients were Japanese; this is because charcoal is frequently used in Japan for barbecues.

It is noteworthy that the patients under 60 years of age normally recovered within two to three months (5-7), except for one patient (8) who did not receive hyperbaric oxygen or corticosteroid pulse therapy. In contrast, the patients above 60 years of age had poor prognoses and did not recover completely within six months $(9,10)$. It should be noted, however, that our patient became independent in activities of daily living within six months, and her cognitive function recovered almost completely within 14 months. Therefore, a follow-up study lasting over one year is needed to determine the prognosis of patients with subacute dementia caused by $\mathrm{CO}$ poisoning.

Another important point is that most of the patients received hyperbaric oxygen or corticosteroid pulse therapy. Although the efficacy of chronic-stage hyperbaric oxygen therapy is debatable, all patients undergoing hyperbaric oxygen recovered to various extents, whereas one patient under 60 years of age who was not given hyperbaric oxygen or corticosteroid pulse therapy remained mildly demented at four months after onset (8). It is notable that hyperbaric oxygen was administered as late as 50 days after disease onset (6). Since the immediate effect of hyperbaric oxygen therapy is limited and the recovery process is slow and lasts 
as long as one year, as observed in the present patient, physicians should administer hyperbaric oxygen even if it is more than one month after disease onset. The number of times therapy should be given and at what interval remain future issues to be clarified.

On the other hand, corticosteroid pulse therapy was administered in one reported patient (5) and our patient, and, in both cases, beneficial effects were evident. The rationale for adopting corticosteroid pulse therapy in the treatment of $\mathrm{CO}$ poisoning is that the delayed leukoencephalopathy results not only from ischemic changes, but also secondary demyelination (11) and inflammation involving myelin basic protein (12). This phenomenon is reflected in a high level of myelin basic protein in the CSF (5). Therefore, it is reasonable to administer corticosteroid pulse therapy while the level of myelin basic protein in the CSF remains high.

In conclusion, for the treatment of subacute dementia caused by $\mathrm{CO}$ poisoning, both hyperbaric oxygen and corticosteroid pulse therapy should be considered. Hyperbaric oxygen should be administered even beyond one month after disease onset, and corticosteroid pulse therapy should be given while the CSF myelin basic protein level is elevated. The use of corticosteroid pulse therapy for the treatment of $\mathrm{CO}$ intoxication has been reported only in Japan $(5,13)$; therefore, further studies are needed to establish its efficacy.

The authors state that they have no Conflict of Interest (COI).

\section{Acknowledgement}

We are grateful to Dr. Tanaka, Department of Neurology, Kanazawa Medical University for assaying the anti-NMDA receptor antibodies and Dr. Chiba, Department of Neurology, Kyorin University for assaying the antibodies associated with paraneoplastic neurological syndromes.

\section{References}

1. Choi IS. Delayed neurologic sequelae in carbon monoxide intoxication. Arch Neurol 40: 433-435, 1983.

2. Ernst A, Zibrak JD. Carbon monoxide poisoning. N Engl J Med 339: 1603-1608, 1998.
3. Doody RS, Jankovic J. The alien hand and related signs. J Neurol Neurosurg Psychiatry 55: 806-810, 1992.

4. Mori E, Yamadori A. Compulsive manipulation of tools and pathological grasp phenomenon. Rinsho Shinkeigaku (Clinical Neurology) 22: 329-335, 1982 (in Japanese, Abstract in English).

5. Abe Y, Tanno Y, Ishihara T, et al. High-dose intravenous methylprednisolone for delayed leuckoencephalopathy after carbon monoxide poisoning: a case report. Shinkei Chiryogaku (Neurological Therapeutics) 26: 625-631, 2009 (in Japanese).

6. Hiroi A, Iijima M, Uchiyama S, Iwata $M$. A case of interval form of carbon monoxide poisoning, who responded to hyperbaric oxygen therapy. Shinkei Naika (Neurological Medicine) 64: 626-632, 2006 (in Japanese, Abstract in English).

7. Sunami Y, Sugaya K, Matsubara S, Yagishita A, Hayashi S. A case of fifty-seven year-old-man suspected of intermittent-type carbon monoxide poisoning on imaging with successful hyperbaric oxygen tehrapy. Tokyo-to Ishikai Zasshi (The Journal of Tokyo Medical Assosiation) 62: 46-48, 2009 (in Japanese).

8. Yamazaki T, Shii H, Kira J. A case of interval form of carbon monoxide poisoning without acute intoxication symptom complicating the diagnosis: differential diagnosis of acute-onset dementia. Ninchi Shinkei Kagaku (Japanese Journal of Cognitive Neuroscience) 14: 193-197, 2013 (in Japanese).

9. Imanaka A, Goami K, Shinno H. A clinical examination into the cases of interval form of carbon monoxide poisoning treated with hyperbaric oxygen therapy. Rinsho Seishin Igaku (Jpn J Clin Psychiatry) 33: 201-207, 2004 (in Japanese).

10. Shiote M, Kido Y, Hayashi T, et al. A case of carbon monoxide intoxication presenting subacute dementia as the initial symptom. Rinsho Shinkeigaku (Clinical Neurology) 42: 212-215, 2002 (in Japanese, Abstract in English).

11. Plum F, Posner J, Hain RF. Delayed neurological deterioration after anoxia. Arch Intern Med 110: 18-25, 1962.

12. Thom SR, Bhopale VM, Fisher D, Zhang J, Gimotty P. Delayed neuropathology after carbon monoxide poisoning is immunemediated. Proc Natl Acad Sci U S A 101: 13660-13665, 2004.

13. Iwamoto K, Ikeda $K$, Mizumura $S$, Tachiki K, Yanagihashi M, Iwasaki Y. Combined treatment of methylprednisolone pulse and memantine hydrochloride prompts recovery from neurological dysfunction and cerebral hypoperfusion in carbon monoxide poisoning: A case report. J Stroke Cerebrovasc Dis 23: 592-595, 2014.

14. Matsuda H, Mizumura S, Soma T, Takemura N. Conversion of brain SPECT images between different collimators and reconstruction processes for analysis using statistical parametric mapping. Nucl Med Commun 25: 67-74, 2004.

15. Friston KJ, Holmes AP, Worsley KJ, Poline J-P, Frith CD, Frackowiak RSJ. Statistical parametric maps in functional imaging: a general linear approach. Hum Brain Mapp 2: 189-210, 1995.

(C) 2014 The Japanese Society of Internal Medicine http://www.naika.or.jp/imonline/index.html 